\title{
Relation between GDOP and the Geometry of the Satellite Constellation
}

\author{
Nuria Blanco-Delgado \\ Centre de Tecnologia Aeroespacial \\ 08840 Viladecans (Barcelona), Spain \\ Email: nuria.blanco@ctae.org
}

\author{
Fernando D. Nunes \\ Instituto de Telecomunicacoes \\ Instituto Superior Tecnico \\ 1049-001 Lisbon, Portugal \\ Email: fernando.nunes@1x.it.pt
}

\author{
Gonzalo Seco-Granados \\ Telecommunications and Systems Engineering \\ Department, Universitat Autonoma de Barcelona \\ 08193 Bellaterra (Barcelona), Spain \\ Email: gonzalo.seco@uab.cat
}

\begin{abstract}
The relationship between Geometrical Dilution Of Precision (GDOP) and maximum volume ${ }^{1}$ of the polytope ${ }^{2}$ expanded by the user-satellite unit vectors endpoints has been used for long as an approach to reduce the time that a receiver devotes to the satellite selection process. Although receivers are able to track all satellites in view, a satellite selection process may still be needed for some applications or when the number of satellites available is large (i.e., due to the availability of several constellations of satellites). This paper evaluates the relationship between the determinant of the GDOP matrix and the maximum volume of the polytope expanded by the user-satellite unit vectors endpoints when any number of satellites is employed for the computation of the position.
\end{abstract}

\section{INTRODUCTION}

Along decades, it has been largely considered that the GDOP is approximately minimized by optimizing the geometry of the satellites [2, ch. 5]. Note that GDOP = $\left\{\operatorname{tr}\left[\left(\boldsymbol{G}^{\mathrm{T}} \boldsymbol{G}\right)^{-1}\right]\right\}^{1 / 2}$ where

$$
\operatorname{tr}\left[\left(\boldsymbol{G}^{\mathrm{T}} \boldsymbol{G}\right)^{-1}\right]=\frac{1}{\operatorname{det}\left(\boldsymbol{G}^{\mathrm{T}} \boldsymbol{G}\right)} \sum_{i i} g_{i i}^{\prime}
$$

and $g_{i j}^{\prime}$ are the elements of the adjoint matrix of $\boldsymbol{G}^{\mathrm{T}} \boldsymbol{G}$. The matrix $G$ is known as the geometry matrix since it characterizes the satellite-user geometry, i.e.,

$$
\boldsymbol{G}=\left[\begin{array}{cccc}
-d_{1 x} & -d_{1 y} & -d_{1 z} & 1 \\
-d_{2 x} & -d_{2 y} & -d_{2 z} & 1 \\
\vdots & \vdots & \vdots & \vdots \\
-d_{h x} & -d_{h y} & -d_{h z} & 1
\end{array}\right]
$$

where $h$ is the number of satellites being tracked by the receiver and $\boldsymbol{d}_{i}=\left(d_{i x}, d_{i y}, d_{i z}\right)$ is the estimated line-of-sight unit vector from the user to the satellite $i$.

Thus, as the adjoint varies less strongly with the geometry than does the determinant ${ }^{3}$ of $\left(\boldsymbol{G}^{\mathrm{T}} \boldsymbol{G}\right)$, minimization of GDOP

\footnotetext{
${ }^{1}$ To maintain the nomenclature, volume is used in $\mathbb{R}^{2}$ referring to area.

${ }^{2} \mathrm{~A}$ polytope is a finite convex region of $n$-dimensional space enclosed by a finite number of hyperplanes [1, pg. 126].

${ }^{3} \operatorname{det}\left(\boldsymbol{G}^{\mathrm{T}} \boldsymbol{G}\right)=\operatorname{det}(\boldsymbol{G})^{2}$ when $\boldsymbol{G} \in \mathbb{R}^{n \times n}$.
}

can be approximated by the maximization of the determinant [2, ch. 5]. Although, ideally, satellite selection should be based on the minimization of the GDOP parameters, this approach is very convenient from a computational point of view since there are efficient algorithms to analyze 2-dimensional (2-D) and 3 dimensional (3-D) geometries. Based on this approximation and the fact that the volume of the $n$-dimensional parallelepiped expanded by the columns of a matrix, $\boldsymbol{A} \in \mathbb{R}^{m \times n}$ with $m \geqslant n$, is $V_{n}=\left[\operatorname{det}\left(\boldsymbol{A}^{\mathrm{T}} \boldsymbol{A}\right)\right]^{1 / 2}$ [3, Example 6.1.4], research has focused on minimizing GDOP by maximizing the volume of the tetrahedron expanded by the user-satellite unit vectors endpoints. In fact, it was shown in [4] that for $h=4$ satellites, the volume of the tetrahedron equals $\operatorname{det}(\boldsymbol{G}) / 6$.

Various publications discuss the relationship between GDOP and the volume of the tetrahedron defined by the usersatellite-unit vectors endpoints, such as [5], [6], [7], [8], and [9]. Therefore, several satellite selection methods have been developed during the last decades based on the correspondence between GDOP minimization and the maximization of the polytope volume [4], [9], [10], [11], [12], and [13], for more than three satellites in 2-D positioning and more than four satellites in 3-D positioning.

However, this correspondence requires that the volume is expanded by the column vectors of a $m \times n$ matrix with $m \geqslant n$. In our case, the satellite-user unit vectors are the row vectors of matrix $\boldsymbol{G}$. Consequently, this only holds for three satellites in 2-D positioning and four satellites in 3-D positioning for which matrix $\boldsymbol{G}$ is square, i.e., $\operatorname{det}(\boldsymbol{G})=\operatorname{det}\left(\boldsymbol{G}^{\mathrm{T}}\right)$. The equivalence between maximum volume and minimum GDOP for five satellite in 2-D positioning is demonstrated for the optimal case, i.e., the regular polytope, in [2, ch. 5]. The purpose of this paper is to evaluate the relationship between maximization of the volume expanded by the user-satellite unit vectors and maximization of $\operatorname{det}\left(\boldsymbol{G}^{\mathrm{T}} \boldsymbol{G}\right)$ in the general case, i.e., for any number of satellites.

The paper is organized as follows. The GDOP concept is reviewed in Section II. The relationship between the determi- 
nant of the geometry matrix, $\boldsymbol{G}$, and the maximum volume polytope defined by the user-satellite unit vectors endpoints for any number of satellites is studied in Section III. Finally, conclusions are drawn in Section IV.

\section{GDOP CONCEPT}

To determine its position, a navigation receiver computes its distance to several satellites. The receiver computes the distance by measuring the time, $\delta t$, it takes to a satellitegenerated signal to reach the receiver antenna. As satellite and receiver clocks are not synchronized, the measured distance is called pseudorange. The pseudorange is given by $\delta t$ multiplied by the speed of light in the vacuum, i.e., $\rho=c \cdot \delta t$. Thus the measured pseudorange between a user and a satellite $i$ can be related to the user position and clock offset as follows:

$$
\rho_{i}=\left\|\boldsymbol{r}_{i}-\boldsymbol{r}_{u}\right\|+b_{u}+\epsilon_{\rho_{i}}, \quad i=1,2, \ldots, h
$$

where $\boldsymbol{r}_{i}=\left(x_{i}, y_{i}, z_{i}\right)$ is the satellite position at the transmit time, $\boldsymbol{r}_{u}=\left(x_{u}, y_{u}, z_{u}\right)$ is the receiver position at the receive time, $b_{u}$ is the bias of the receiver clock in meters, and $\epsilon_{\rho_{i}}$ is the composite of errors produced by satellite ephemerides and atmospheric delays mismodeling, receiver noise, etc.

The pseudorange residual error relative to satellite $i$ (i.e., $\Delta \rho_{i}$ ) which is the difference between the predicted $\hat{\rho}_{i}$ and the actual $\rho_{i}$, can be linearly related to the error in the state estimate, $\boldsymbol{\Delta} \boldsymbol{x} \equiv\left[\boldsymbol{\Delta} \boldsymbol{r}^{\mathrm{T}} \Delta b\right]^{\mathrm{T}}$, by performing a Taylor series expansion about the current state estimate. Only first order partial derivatives are considered, resulting in,

$$
\Delta \rho_{i}=\left[-\boldsymbol{d}_{i}^{\mathrm{T}} 1\right] \boldsymbol{\Delta} \boldsymbol{x}+\Delta \epsilon_{\rho_{i}}
$$

where

$$
\begin{aligned}
& \boldsymbol{d}_{i} \equiv \frac{\boldsymbol{r}_{i}-\widehat{\boldsymbol{r}}_{u}}{\left\|\boldsymbol{r}_{i}-\hat{\boldsymbol{r}}_{u}\right\|}, \Delta \boldsymbol{r} \equiv \widehat{\boldsymbol{r}}_{u}-\boldsymbol{r}_{u}, \\
& \Delta b \equiv \widehat{b}_{u}-b_{u}, \text { and } \Delta \epsilon_{\rho_{i}}=\hat{\epsilon}_{\rho_{i}}-\epsilon_{\rho_{i}}
\end{aligned}
$$

$\Delta r$ is the residual error in the estimation of the receiver position, $\Delta b$ is the residual error in the estimation of the receiver clock bias in meters, $\Delta \epsilon_{\rho_{i}}$ is the residual error after the known biases from satellite $i$ have been removed.

The navigation equations can be put in matricial form by defining the vectors $\boldsymbol{\Delta} \boldsymbol{\rho}=\left[\Delta \rho_{1}, \Delta \rho_{2}, \ldots, \Delta \rho_{h}\right]^{\mathrm{T}}$ and $\Delta \epsilon_{\rho}=\left[\Delta \epsilon_{\rho_{1}}, \Delta \epsilon_{\rho_{2}}, \ldots, \Delta \epsilon_{\rho_{h}}\right]^{\mathrm{T}}$ yielding

$$
\Delta \rho=G \Delta x+\Delta \epsilon_{\rho} .
$$

Assuming that the measurement errors $\Delta \epsilon_{\rho_{i}}$ are independent and identically Gaussian distributed zero-mean and variance $\sigma^{2}$, the least-squares solution for general $h \geqslant 4$ (in 3-D positioning) or $h \geqslant 3$ (in 2-D positioning) is [14, pp. 329]

$$
\boldsymbol{\Delta} \widehat{\boldsymbol{x}}=\left(\boldsymbol{G}^{\mathrm{T}} \boldsymbol{G}\right)^{-1} \boldsymbol{G}^{\mathrm{T}} \boldsymbol{\Delta} \boldsymbol{\rho} .
$$

Note that in the case of 2-D positioning the third component of the user position $d_{i z}$ is assumed to be perfectly known, i.e., it is not considered as an unknown in the navigation equations (3). Therefore, matrix $\boldsymbol{G}$ has one column less.

The accuracy of this least-squares solution is decided by two factors, i.e., the quality of the measurements and the userto-satellite geometry. The measurement quality is described by $\sigma^{2}$, whereas the geometry is described by matrix $\boldsymbol{G}$. The covariance of position, under the stated assumptions on the distribution of the measurement errors, is given by

$$
\mathrm{E}\left[\boldsymbol{\Delta} \hat{\boldsymbol{x}} \boldsymbol{\Delta} \hat{\boldsymbol{x}}^{\mathrm{T}}\right]=\sigma^{2}\left(\boldsymbol{G}^{\mathrm{T}} \boldsymbol{G}\right)^{-1}
$$

Matrix $\left(\boldsymbol{G}^{\mathrm{T}} \boldsymbol{G}\right)^{-1}$ is known as the GDOP matrix. The scalar parameter GDOP is defined as the square root of the trace of the GDOP matrix, i.e.,

$$
\mathrm{GDOP}=\frac{1}{\sigma}\left[\sigma_{x_{u}}^{2}+\sigma_{y_{u}}^{2}+\sigma_{z_{u}}^{2}+\sigma_{b_{u}}^{2}\right]^{1 / 2}
$$

In selecting the satellites, the GDOP values should be as small as possible in order to generate the best user position accuracy. Several parameters are typically employed to characterize the time/position solution derived from GDOP: position dilution of precision $\left(\mathrm{PDOP}=\sigma^{-1}\left[\sigma_{x_{u}}^{2}+\sigma_{y_{u}}^{2}+\sigma_{z_{u}}^{2}\right]^{1 / 2}\right.$ ), horizontal dilution of precision (HDOP $=\sigma^{-1}\left[\sigma_{x_{u}}^{2}+\right.$ $\left.\sigma_{y_{u}}^{2}\right]^{1 / 2}$ ), vertical dilution of precision (VDOP $=\sigma^{-1} \sigma_{z_{u}}$ ), and time dilution of precision ( $\mathrm{TDOP}=\sigma^{-1} \sigma_{b_{u}}$ ).

\section{DETERMinANT MAXimization Through Volume MAXIMIZATION}

For three satellites in 2-D and four satellites in 3-D, there is a direct relationship between determinant and volume [15, ch. 9 sec. 5]. However, when more than three or four satellites are to be selected, the relationship is not direct any more [15, ch. 9 sec. 5]. In this section, we analyze the relationship between $\operatorname{det}\left(\boldsymbol{G}^{\mathrm{T}} \boldsymbol{G}\right)$ and the volume of the polytope formed by the user-satellite unit vectors endpoints.

Let us review first what happens when three satellites are employed for the computation of a 2-D position (or four satellites in 3-D). Three satellites are needed to obtain a position estimate in a 2-D coordinate system. The clock synchronization error is accounted for as an unknown in the linearized navigation equation (4). Therefore, the geometry matrix is

$$
\boldsymbol{G}=\left[\begin{array}{lll}
-d_{1 x} & -d_{1 y} & 1 \\
-d_{2 x} & -d_{2 y} & 1 \\
-d_{3 x} & -d_{3 y} & 1
\end{array}\right] .
$$

The addition of the column of ones results in the determinant of $\boldsymbol{G}$ matching the area of the parallelogram formed by the user-satellite unit vectors endpoints when taking one of 
them as a vertex. That is,

$$
\begin{aligned}
|\operatorname{det}(\boldsymbol{G})| & =|\boldsymbol{a} \times \boldsymbol{b}| \\
& =\left|\operatorname{det}\left[\begin{array}{ll}
\left(d_{2 x}-d_{1 x}\right) & \left(d_{3 x}-d_{1 x}\right) \\
\left(d_{2 y}-d_{1 y}\right) & \left(d_{3 y}-d_{1 y}\right)
\end{array}\right]\right|
\end{aligned}
$$

where $\boldsymbol{a}=\boldsymbol{d}_{2}-\boldsymbol{d}_{1}$ and $\boldsymbol{b}=\boldsymbol{d}_{3}-\boldsymbol{d}_{1}, \boldsymbol{d}_{i}=\left(d_{i x}, d_{i y}\right)$, $i=1,2$ and $3, \times$ denotes the cross product, and $|\cdot|$ denotes absolute value. The situation is illustrated graphically in Fig. 1.

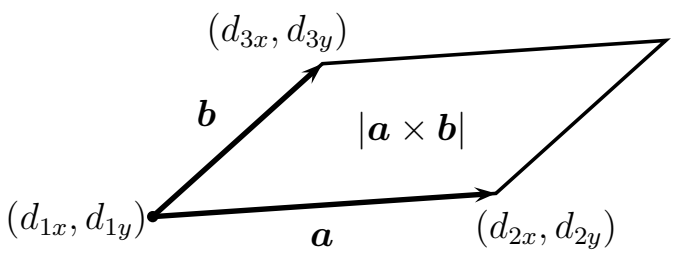

Fig. 1. Area of a parallelogram as a cross-product.

Thus, the area of the polygon formed by those points is equal to half the determinant of the geometry matrix, $\pm 1 / 2 \operatorname{det}(\boldsymbol{G})$, [3, Example 5.13.2], [16]. The \pm is meant to take whichever sign is needed so that the result is positive (non-negative); a minus meaning that the order of the vectors is not counterclockwise. The same applies when selecting four satellites to obtain a 3 -D position. In that case $\operatorname{det}(\boldsymbol{G})$ is proportional to the volume of the tetrahedron - specifically, $\operatorname{det}(\boldsymbol{G})= \pm 6 \cdot V_{n}[16]$.

The previous conclusions are a consequence of the property of the determinant whereby row substraction leaves the determinant unchanged. But this is only valid for a square matrix. Therefore, one could argue that this is true only for the cases presented here of selecting three satellites in 2-D or four satellites in 3-D positioning. When $h$ is the number of satellites to be considered for the position computation, the geometry matrix is $\boldsymbol{G}_{h \times 4}$,

$$
\boldsymbol{G}_{h \times 4}=\left[\begin{array}{cccc}
-d_{1 x} & -d_{1 y} & -d_{1 z} & 1 \\
-d_{2 x} & -d_{2 y} & -d_{2 z} & 1 \\
\vdots & \vdots & \vdots & \vdots \\
-d_{h x} & -d_{h y} & -d_{h z} & 1
\end{array}\right] .
$$

If $\boldsymbol{A} \in \mathbb{R}^{n \times n}$, then $V_{n}=|\operatorname{det}(\boldsymbol{A})|$ [3, Example 5.13.2]. Furthermore, if $\boldsymbol{A} \in \mathbb{R}^{m \times n}$ with $m \geqslant n, V_{n}=$ $\left[\operatorname{det}\left(\boldsymbol{A}^{\mathrm{T}} \boldsymbol{A}\right)\right]^{1 / 2}$ is proportional to the volume of the $n$ dimensional parallelepiped expanded by the columns of $\boldsymbol{A}$ [3, Example 6.1.4]. However, the volume we are interested in is not the one spanned by the columns of $\boldsymbol{A}$ but the one expanded by its rows. Therefore, the relationship between the determinant of the GDOP matrix and the volume expanded by its row vectors is analyzed next.

The relationship between the volume expanded by the satellite-user unit vectors endpoints and $\operatorname{det}\left(\boldsymbol{G}^{\mathrm{T}} \boldsymbol{G}\right)$ for more than four satellites in 3-D or more than three satellites in 2-D is not direct, as stated before. However, what actually occurs when having a larger number of satellites has not been evaluated. A Monte Carlo simulation of $10^{5}$ runs was performed, where a geometry matrix was generated with random unitary vectors $\boldsymbol{d}_{i} \in \mathbb{R}^{2}$. The area determined by the set of vectors $\boldsymbol{d}_{i}$ and the determinant, $1 / 2\left[\operatorname{det}\left(\boldsymbol{G}^{\mathrm{T}} \boldsymbol{G}\right)\right]^{1 / 2}$, were obtained. This process was repeated for several amounts of satellites. Results for $h=3,4,5$ and 6 satellites in $\mathbb{R}^{2}$ are plotted in Figs. 2(a), (b), (c), and (d), respectively. Area denotes the area of the polygon formed by the endpoint vectors and Determinant denotes $1 / 2\left[\operatorname{det}\left(\boldsymbol{G}^{\mathrm{T}} \boldsymbol{G}\right)\right]^{1 / 2}$.

When $h=3$, Fig. 2(a), there is a direct relationship Determinant-Area, in agreement with Equation (11). But, when the number of satellites increases, the relationship is not direct anymore and the points are spread over a region, i.e., the blue dots in Fig. 2. The upper line in Figs. 2(b), (c), and (d), corresponds to a regular polygon whose number of sides is determined by the area value. That is, considering that the area of a regular polygon of $l$ sides inscribed in the unit circle is given by [17, pg.111],

$$
\text { Area }=l \cos \left(\frac{180}{l}\right) \sin \left(\frac{180}{l}\right),
$$

the upper line of each plot corresponds to drawing a geometric figure that is the closest to a regular polygon for that area value. Thus, several regions can be identified in Fig. 2(b), (c) and (d); these regions correspond to the value of the area for the different regular polygons that can be formed. They are delimited by the red vertical lines in Fig. 3 . That is, $0 \leqslant$ Area $\leqslant 1.299$ for a triangle, $1.299<$ Area $\leqslant 2$ for a quadrilateral, $2<$ Area $\leqslant 2.3776$ for a pentagon, and $2.3776<$ Area $\leqslant$ 2.5981 for a hexagon.

To illustrate this, the geometric figures resulting from similar values of the area and the two extreme values of the determinant are plotted in Figs. 3 and 4 for $h=5$ and $h=6$ in $\mathbb{R}^{2}$, respectively. Fig. 3(a) and (b) correspond to the equilateral triangle region, i.e., Area $=1.299$, and Fig 3(c) and (d) to the regular quadrilateral region, i.e., Area $=2$. Similar results are plotted in Fig. 4 for $h=5$ to show that the same happens for any $h$ at the same Area values. Note that the set giving the smallest GDOP will be the one that expands a geometric figure that is closer to the corresponding regular polyhedron, i.e., the one with the largest determinant value.

In conclusion, there is a relationship between the largest volume polytope that can be formed and the minimum GDOP (following the statement in [2, ch. 5]) when more than three satellites are considered in $\mathbb{R}^{2}$ or more than four in $\mathbb{R}^{3}$. However, apart from volume value, satellite disposition should also be considered; giving priority to those closer to a regular 


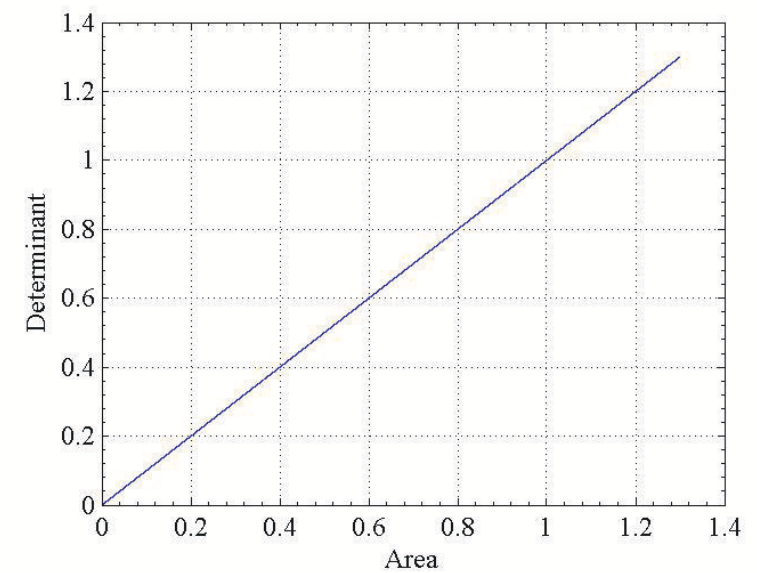

(a)

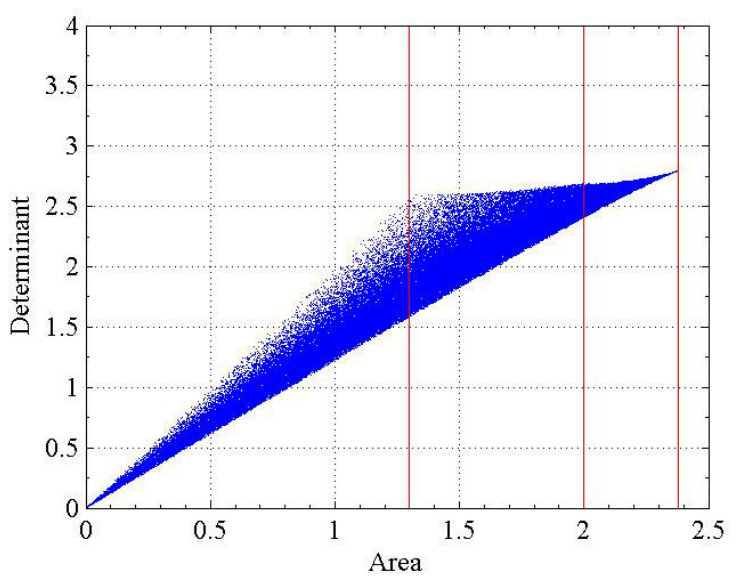

(c)

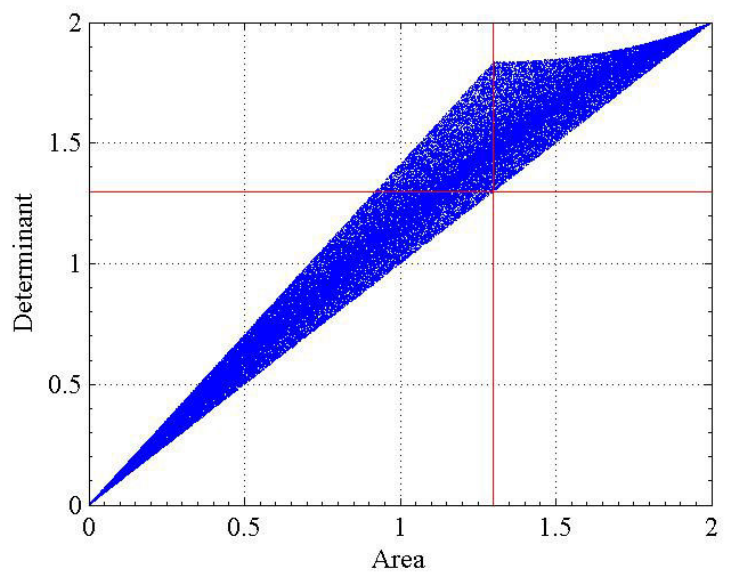

(b)

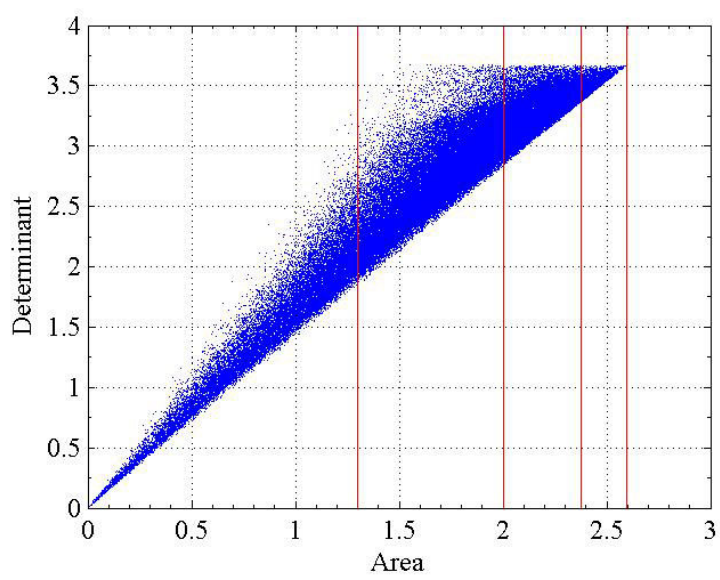

(d)

Fig. 2. Discrepancy plots of determinant versus the volume for all possible solutions in $\mathbb{R}^{2}$. (a) $h=3$. (b) $h=4$. (c) $h=5$. (d) $h=6$.

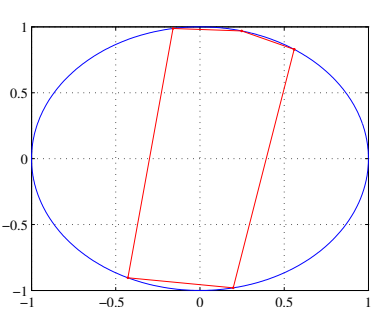

(a)

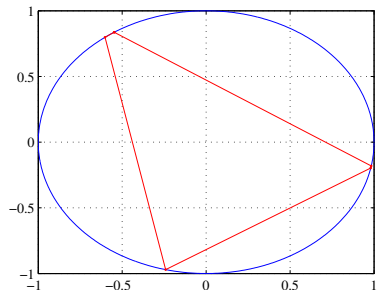

(b)

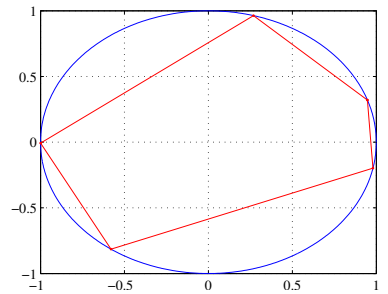

(c)

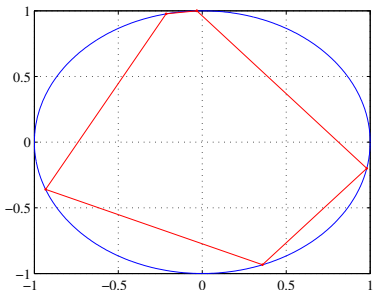

(d)

Fig. 3. Areas generated at several points of Fig. 2 (c), i.e., with $h=5$ in $\mathbb{R}^{2}$. (a) Area $\approx 1.299$ and Determinant $\approx 1.65$. (b) Area $\approx 1.299$ and Determinant $\approx 2.6$. (c) Area $\approx 2$ and Determinant $\approx 2$.4. (d) Area $\approx 2$ and Determinant $\approx 2.7$.

polytope. For example, for each of the regions identified before, we should search for the set of $l$ satellites whose geometry is closer to a regular polyhedron with equal number of satellites in all its vertices. Thus, the volume can be considered as an indicative of the disposition of the satellites that the receiver needs to track in order to get a specific performance in terms of GDOP.

This is exemplified in Fig. 5 for $h=5$ in $\mathbb{R}^{2}$ and Area $\approx 1.299$, i.e., for an equilateral triangle. The area of Fig. 5(a) is larger than that of Fig. 5(b) while the determinant is the other way round. It can be seen that, even if the area is smaller in Fig. 5(b), the determinant is larger and, thus, the GDOP value is smaller. This supports the statement that, for each of the regions delimited by the volume of the polygon, the figure providing the smallest GDOP is the one whose geometry is closer to the one of the corresponding regular polygon. 


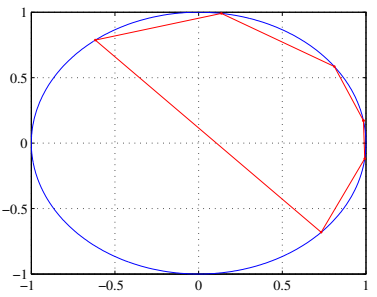

(a)

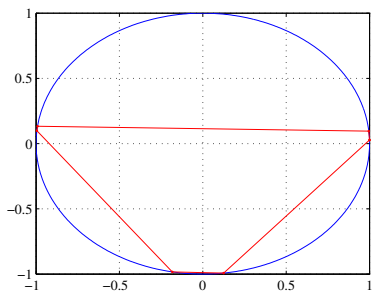

(b)

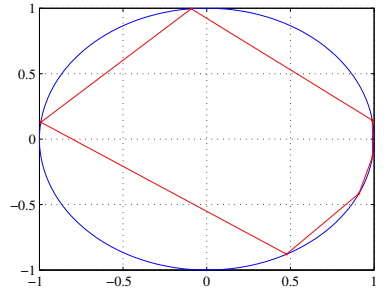

(c)

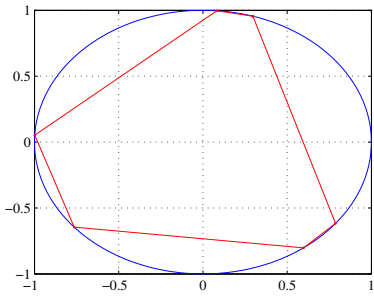

(d)

Fig. 4. Areas generated at several points of Fig. 2 (d), i.e., with $h=6$ in $\mathbb{R}^{2}$. (a) Area $\approx 1.299$ and Determinant $\approx 1.65$. (b) Area $\approx 1.299$ and Determinant $\approx 2.6$. (c) Area $\approx 2$ and Determinant $\approx 2.4$. (d) Area $\approx 2$ and Determinant $\approx 2.7$

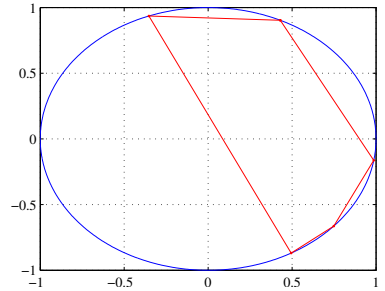

(a)

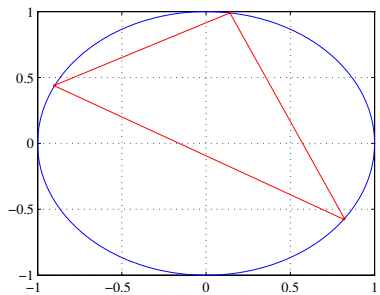

(b)

Fig. 5. Areas generated at several points of Fig. 2 (c), i.e., with $h=5$ in $\mathbb{R}^{2}$. (a) Area $\approx 1.2$ and Determinant $\approx 1.5$. (b) Area $\approx 1.0$ and Determinant $\approx$ 2.0 .

\section{Conclusions And Significance}

The discrepancy between maximization of the determinant of the GDOP matrix and the volume defined by the satelliteuser-unit vectors was evaluated. Results show that both functions have a very similar behavior for large volumes and thus, the error can be considered negligible. However, only when the optimal solution corresponding to a regular polytope is present can we assure that the solution provided is the optimal one, i.e., the one that minimizes GDOP. Note that the larger the number of satellites in view is, the more likely a regular polytope volume can be obtained. Therefore, the possibility of choosing a geometry close to the one of a regular polytope increases and so it does choosing a GDOP value closer to the optimal one.

It was also concluded that the approximation between maximum volume defined by the user-to-satellite unit vectors endpoints and minimization of the error due to the usersatellite geometry is also valid when more than three or four satellites are to be selected in 2-D and 3-D positioning, respectively. However, other aspects must be considered along with volume maximization; i.e., the number of satellites $l$ to track should be those whose geometry is closer to a regular polyhedron with equal number of satellites in all its vertices.

Summarizing, the value of the volume can be considered as an indicative of the optimal disposition of the satellites that will result in a minimal value of GDOP.

\section{REFERENCES}

[1] H. S. M. Coxeter, Regular Polytopes. Methuen and Co., 1948.
[2] B. Parkinson and J. Spilker, The Global Positioning System : Theory and Applications, ser. Progress in astronautics and aeronautics. Washington, DC: American Institute of Aeronautics and Astronautics, 1996, vol. I.

[3] C. D. Meyer, Matrix Analysis and Applied Linear Algebra. Society for Industrial \& Applied Mathematics, 2001.

[4] M. Kihara and T. Okada, "A Satellite Selection Method and Accuracy for the Global Positioning System," Journal of the Institute of Navigation, vol. 31, no. 1, pp. 8-20, 1984.

[5] R. Yarlagadda, I. Ali, N. Al-Dhahir, and J. Hershey, "GPS GDOP metric," in IEE Proceedings Radar, Sonar and Navigation, October 2000, pp. 259-264.

[6] A. Phillips, "Geometrical Determination of PDOP," Journal of the Institute of Navigation, vol. 31, no. 4, pp. 329-337, Winter 1984-85.

[7] P. Massatt and K. Rudnick, "Geometric Formulas for Dilution of Precision Calculations," Journal of the Institute of Navigation, vol. 37, no. 4, pp. 379-391, Winter 1990-91.

[8] D. Hsu, "Relations Between Dilutions of Precision and Volume of the Tetrahedron Formed by Four Satellites," in Proceedings of the IEEE/ION Position, Location and Navigation Symposium, PLANS 1994, Las Vegas, NV, April 1994, pp. 669-676.

[9] Z. Zheng, C. Huang, C. Feng, and F. Zhang, "Selection of GPS Satellites for the Optimum Geometry," Chinese Astronomy and Astrophysics, vol. 28, no. 1, pp. 80-87, 2004.

[10] Z. Miaoyan and Z. Jun, "A Fast Satellite Selection Agorithm: Beyond Four Satellites," IEEE Journal of Selected Topics in Signal Processing, vol. 3, no. 5, pp. 740-747, October 2009.

[11] J. Li, A. Ndili, L. L. Ward, and S. Buchman, "GPS Receiver Satellite/Antenna Selection Algorithm for the Stanford Gravity Probe B Relativity Mission," in Proceddings of the National Technical Meeting of the Institute of Navigation, ION NTM 1999, San Diego, CA, January 1999, pp. 541-550.

[12] D. Roongpiboonsopit and H. Karimi, "A Multi-Constellations Satellite Selection Algorithm for Integrated Global Navigation Satellite Systems," Journal of Inteligent Transportation Systems, vol. 13, no. 3, pp. 127$141,2009$.

[13] N. Blanco-Delgado and F. Nunes, "A Satellite Selection Method for Multi-Constellation GNSS Using Convex Geometry," IEEE Trans. Veh. Technol., vol. 59, no. 9, pp. 4289-4297, November 2010. 
[14] J. Gentle, Matrix Algebra: Theory, Computations and Applications in Statistics. Springer, 2007.

[15] F. Gantmacher, The Theory of Matrices. AMS Chelsea Publishing, 1960, vol. I.

[16] H. Newson, "On the Volume of a Polyhedron," The Annals of Mathematics, vol. 1, no. 1/4, pp. 108-110, 1899-1900.

[17] I. Niven, Maxima and Minima Without Calculus. The Mathematical Association of America, 1981, vol. Dolciani Mathematical Expositions No.6. 